\title{
An indelible lineage marker for Xenopus using a mutated green fluorescent protein
}

\author{
M. Zernicka-Goetz ${ }^{1, *}$, J. Pines ${ }^{2}$, K. Ryan ${ }^{2}$, K. R. Siemering ${ }^{3}$, J. Haseloff ${ }^{3}$, M. J. Evans ${ }^{1}$ and J. B. Gurdon ${ }^{2}$ \\ Wellcome/CRC Institute, Tennis Court Road, Cambridge CB2 1QR, UK and Departments of ${ }^{2}$ Zoology and ${ }^{1}$ Genetics, University of \\ Cambridge, MRC Laboratory of Molecular Biology, Hills Road, Cambridge, UK \\ ${ }^{*}$ On leave from Department of Embryology, Institute of Zoology, University of Warsaw, Krakowskie Przedmiescie 26/28, 00-927 Warsaw, Poland
}

\section{SUMMARY}

We describe the use of a DNA construct (named GFP.RN3) encoding green fluorescent protein as a lineage marker for Xenopus embryos. This offers the following advantages over other lineage markers so far used in Xenopus. When injected as synthetic mRNA, its protein emits intense fluorescence in living embryos. It is non-toxic, and the fluorescence does not bleach when viewed under $480 \mathrm{~nm}$ light. It is surprisingly stable, being strongly visible up to the feeding tadpole stage ( 5 days), and in some tissues for several weeks after mRNA injection. We also describe a construct that encodes a blue fluorescent protein. We exemplify the use of this GFP.RN3 construct for marking the lineage of individual blastomeres at the 32- to 64-cell stage, and as a marker for single transplanted blastula cells. Both procedures have revealed that the descendants of one embryonic cell can contribute single muscle cells to nearly all segmental myotomes rather than predominantly to any one myotome. An independent aim of our work has been to follow the fate of cells in which an early regulatory gene has been temporarily overexpressed. For this purpose, we co-injected GFP.RN3 mRNA and mRNA for the early Xenopus gene Eomes, and found that a high concentration of Eomes results in ectopic muscle gene activation in only the injected cells. This marker may therefore be of general value in providing long term identification of those cells in which an early gene with ephemeral expression has been overexpressed.

Key words: Xenopus, green fluorescent protein, marker, Eomes, cell lineage, GFP.RN3

\section{INTRODUCTION}

Many important Xenopus transcription factors are strongly expressed in early development, but cease to be expressed a few hours later (Smith, 1993; Dawid, 1994). To determine the function of such genes, one important experimental route is to express them ectopically in a tissue where they are not normally functional, and to ask if the cell fate is changed. However, most markers of cell fate are expressed only after the time when early regulatory genes have ceased to be so. This makes it difficult to relate the overexpression of such a gene in any precise way to a change of cell fate. Thus, in the absence of a lineage marker, it is not clear whether the cells that overexpress a gene themselves change fate, or whether these cells signal to their neighbours to do so. A long-lasting lineage marker is needed to provide continuing recognition of the cells in which an early, but temporary, overexpression has taken place.

The lineage markers so far used in Xenopus are subject to certain limitations (reviewed by Slack, 1983; Smith et al., 1985; Gardner and Lawrence, 1986). Injected colloidal gold particles (Niehrs and De Robertis, 1991) are suitable for whole-mount analyses but are subject to dilution as cell number increases with growth. Fluorescent lysinated dextrans (e.g. Gimlich and Braun, 1985) are not necessarily distributed to the same blastomeres as the injected mRNAs commonly used to achieve ectopic expression. Furthermore, they become hard to detect as development proceeds, and are unstable to the ultraviolet light required to see them. mRNAs encoding enzymes such as $\beta$-galactosidase and horseradish peroxidase should be equally distributed, but have to be visualized by substrate reactions, which do not always give accurate localization of the enzyme. Most importantly, none of these lineage markers can be seen in living cells. A potentially ideal lineage marker is provided by the green fluorescent protein of Aequorea victoria (Chalfie et al., 1994). However, the green fluorescent protein so far used in Xenopus embryos (Tannahill et al., 1995) gives only a moderate strength of signal, and is not expressed until the neurula stage. Peters et al. (1995) suggest that, due to background yolk fluorescence, GFP fusion proteins will have limited utility in Xenopus. Wu et al. (1995) used a recombinant vaccinia virus containing a GFP gene to infect the brain of 3-day Xenopus tadpoles, but not embryos.

We describe here the use of a modified construct (GFP.RN3) encoding green fluorescent protein; this provides a very long lasting and brilliant fluorescence, far exceeding the low level of autofluorescence of Xenopus embryos. We apply this lineage marker to follow the fate of cells ectopically expressing the Xenopus gene Eomes. Eomes encodes a T-domain transcription factor capable of activating nearly all other early Xenopus 
regulatory genes. However, as indicated above, Eomes transcripts are no longer present by the time when most cell fate markers can be detected (K. Ryan, N. Garrett, A. Mitchell and J. B. Gurdon, unpublished). GFP mRNA, coinjected with Eomes mRNA, enables us to identify those cells in which Eomes was overexpressed, and hence to relate the ectopic expression of Eomes to a particular type of differentiation.

In the course of this work, we have found that the GFP.RN3 construct we have used creates such long lasting and strong fluorescence that it is likely to be a reagent of very wide application (see Discussion). To test its usefulness as a lineage marker, we have labelled single cells of an early blastula to follow lineage, and have transplanted single labelled blastula cells to unlabelled hosts. We have followed the distribution of the lineage descendants of the labelled cells into a range of larval tissues, especially muscle, formed a few days later.

\section{MATERIALS AND METHODS}

\section{GFP and BFP mutagenesis}

The modified GFP constructs were generated from $m g f p 5$. $m g f p 5$ incorporates two mutations that improve the folding of the apoprotein at higher temperatures, and thus enhance the solubility of GFP (K. R. S., R. Golbik, R. Sever and J. H., unpublished observations). This substantially increases the fluorescence signal. A third mutation, I167T (Heim et al., 1994) increases the absorption of GFP in the blue part of the spectrum. In addition, $m g f p 5$ incorporates a number of codon changes that remove a cryptic splice site, which is recognised in plants (J. H., K. R. S., D. Prasher and S. Hodge, unpublished observations), and these changes might also have an effect on mRNA processing and/or translational efficiency in animal cells. Two mutations, F64L and S65T, were introduced into $m g f p 5$ by PCR mutagenesis. The S65T mutation enhances the second peak of absorption (Heim et al., 1995) and the F64L mutation (Cormack et al., 1996) enhances the solubility of GFP, at least when it is expressed in bacteria. Two other mutations, H25Y and G191D, were inadvertantly introduced during PCR mutagenesis and identified by A. Brand and C. Davidson, but have no discernable effect on the fluorescence intensity of our modified GFP (J.P., unpublished observations). The modified BFP construct was also derived from $m g f p 5$. It contains the Y66H (Heim et al., 1994) and Y145F (Heim and Tsien, 1996) mutations that shift the absorption of GFP towards the UV, and the emission into the blue part of the spectrum, plus a third mutation, A87T, whose effect is presently unknown.

The mutated GFP, as encoded by our GFP.RN3 construct, is easily visible with a standard FITC filter set, namely with FITC excitation filters BP 450-490 nm, a $510 \mathrm{~nm}$ dichromatic mirror and a $515 \mathrm{~nm}$ suppression filter. Our BFP is seen with standard DAPI excitation filters BP $355-425 \mathrm{~nm}$, a $455 \mathrm{~nm}$ dichromatic filter and a $470 \mathrm{~nm}$ suppression filter.

\section{RN3 constructs}

The GFP and BFP gene coding regions were PCR-amplified from parental vectors (pCMXF64GFP and pCMXBFP, respectively) and cloned in the vector pBluescript RN3P (a MCS-containing derivative of pBluescript RN3, Lemaire et al., 1995; D. Crease, personal communication) between the BamHI (5') and NotI (3') sites. These genes were modified to contain a Xenopus $\beta$-1-globin ribosome binding site [ACAGCCACC(ATG)] at the $5^{\prime}$ end (K. Ryan, N. Garrett, A. Mitchell and J. B. Gurdon, unpublished) and stop codons in all three reading frames at the $3^{\prime}$ end by inclusion of the appropriate sequences in the PCR primers. The resulting clones were named $p \beta G F P / R N 3 P$ and $\mathrm{p} \beta \mathrm{BFP} / \mathrm{RN} 3 \mathrm{P}$.

\section{Microinjection}

mRNA was synthesized, eggs or embryos injected, and embryos cultured as before (Lemaire et al., 1995).

\section{Histology and antibody staining}

Embryos were fixed for 2 hours in $4 \%$ formaldehyde, dehydrated, embedded in hot wax after xylene treatment, sectioned and stained with antibody (Kintner and Brockes, 1984) as in our previous work (Gurdon et al., 1993a).
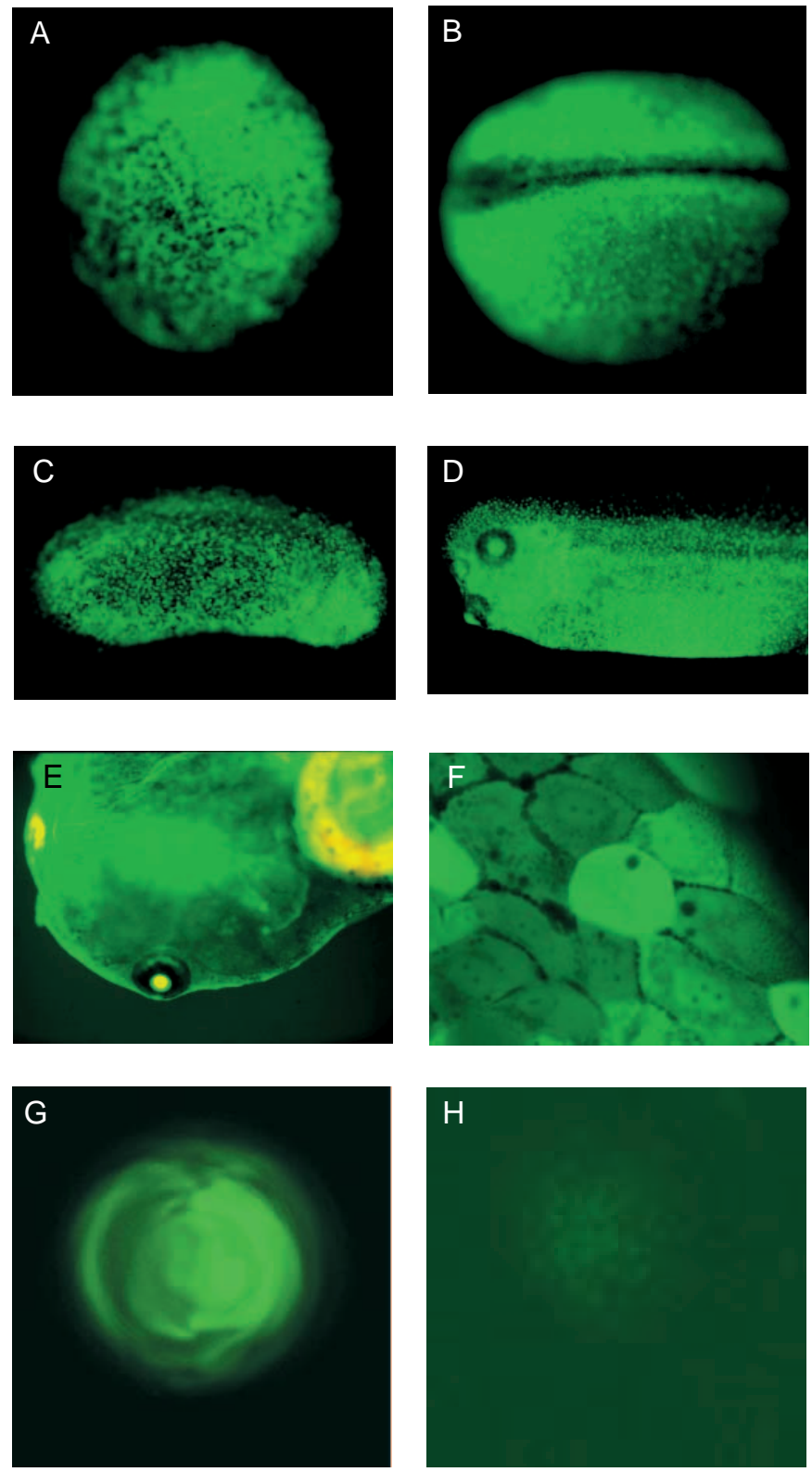

Fig. 1. Whole embryos or larvae labelled by injecting $1 \mathrm{ng}$ GFP.RN3 mRNA into the animal hemisphere of each of the first two blastomeres. (A) Stage 8.5; (B) stage 20; (C) stage 25; (D) stage 37; (E) stage 47; (F) epithelial cells of stage-47 tadpole (all are GFPpositive, but some are brighter than others). (G) Lens of stage-49 tadpole; $(\mathrm{H})$ same view as $\mathrm{G}$, but of unlabelled control tadpole. The black patches in A-D (and also in Fig. 2A-C) are due to pigment. The yellow regions in $\mathrm{E}$ are due either to gut autofluorescence or to the photographic consequence of very high intensity green fluorescence associated with the lens and residual sucker (see legend to Fig. 3D). 


\section{RESULTS}

\section{GFP and BFP constructs}

Compared to the GFP first described (Prasher et al., 1992), a number of amino acid changes have been introduced. These are described in Materials and Methods.

The GFP and BFP sequences have been cloned into the high expression vector bluescript RN3, described by Lemaire et al. (1995). The GFP cDNA RN3 has been further modified by the addition of a ribosome binding site (see Materials and Methods).

\section{Whole embryo labelling}

To test the efficacy of our construct, we injected GFP.RN3 mRNA (1 or $2 \mathrm{ng}$ ) into 2-cell eggs and cultured these as whole embryos until the feeding larval stage. The results shown in Fig. 1 permit the following conclusions. (1) GFP.RN3 expression is strongly visible at stage 8 (Figs 1A, 2A,C), 4 hours after mRNA injection. It is first seen 1.5 hours after injection, when 2-cell embryos have reached the 32-cell stage, at $23^{\circ} \mathrm{C}$. (2) GFP.RN3 is evenly expressed in epidermal cells, the direct descendants of the region of eggs injected with mRNA (Fig. 1A-D). (3) GFP.RN3 fluorescence persists with strong intensity until at least stage 41 ( 80 hours after injection), and can still be detected in stage-53 tadpoles ( 3 weeks after injection, weakly in all cells of the injected lineage but very strongly in the lens, as in Fig. 1E). The longevity of GFP.RN3 as a fluorescent marker may be due to both mRNA and protein stability. The amount of injected GFP.RN3 mRNA starts to decline at stage 18, declines to $24 \%$ of its original level at stage 26 , and to $0.5 \%$ at stage 46 . GFP.RN3 fluorescence is particularly long lasting in those cells which cease division during embryo life, such as lens (Fig. $1 \mathrm{G}, \mathrm{H}$ ) and muscle (Fig. 2I,J). (4) GFP.RN3 fluorescence is sufficiently intense that details of tissue structure can be readily detected by confocal microscopy right through embryogenesis and larval life (Fig. 1F). (5) At least $2 \mathrm{ng}$ per embryo of GFP.RN3 mRNA and its derived protein appear entirely harmless, since injected eggs grow normally to mid-larval life and beyond. Furthermore, frequent viewing of live embryos under $480 \mathrm{~nm}$ light also appears harmless.

\section{Lineage labelling}

Although there are very detailed descriptions of cell lineage from 32-cell Xenopus embryos (Jacobson and Hirose, 1981;

Fig. 2. GFP.RN3- or BFP-labelling of single blastomere lineages. (A,B) $1 \mathrm{ng}$ of GFP.RN3 mRNA injected into one of first two blastomeres; (A) stage 8, animal pole; (B) stage 18, right half of neural plate. (C-F) Injection of a single tier A blastomere at the 32to 64-cell stage with GFP.RN3 mRNA; the descendants of this cell become progressively dispersed; (C) stage 8, animal pole; (D) stage 18 (labelled cells in left-hand neural fold); (E) stage 24; (F) stage 35 (labelled cells in anterior (head) region in $\mathrm{E}$ and $\mathrm{F}$ ). $(\mathrm{G}, \mathrm{H})$ Injection of two adjacent tier A blastomeres on one side of a 16- to 32-cell embryo, one with with BFP, and the other with GFP.RN3; (G) stage 18; (H) stage 26, higher magnification. (I,J) Injection of a single blastomere in tier B at the 32- to 64-cell stage with GFP.RN3 mRNA. Note labelling of individual muscle cells in each segment (I) and in interspersed or alternating segments $(\mathrm{J})$. I and $\mathrm{J}$ both show the tail region of stage- 40 tadpoles (head out of view to the left in I, right in J). The white lines outline the shape of the tail and two single somites containing at least 100 muscle cells.
Dale and Slack, 1987; Moody, 1987; Nakamura and Kishiyama, 1971; Nakamura et al., 1978), little has been reported for later stages (Jacobson, 1983, 1985). Furthermore, all of the lineage markers so far used successfully in Xenopus (see Introduction) have the restriction that only fixed embryos can be analyzed. This means that the same lineage can never be viewed in one
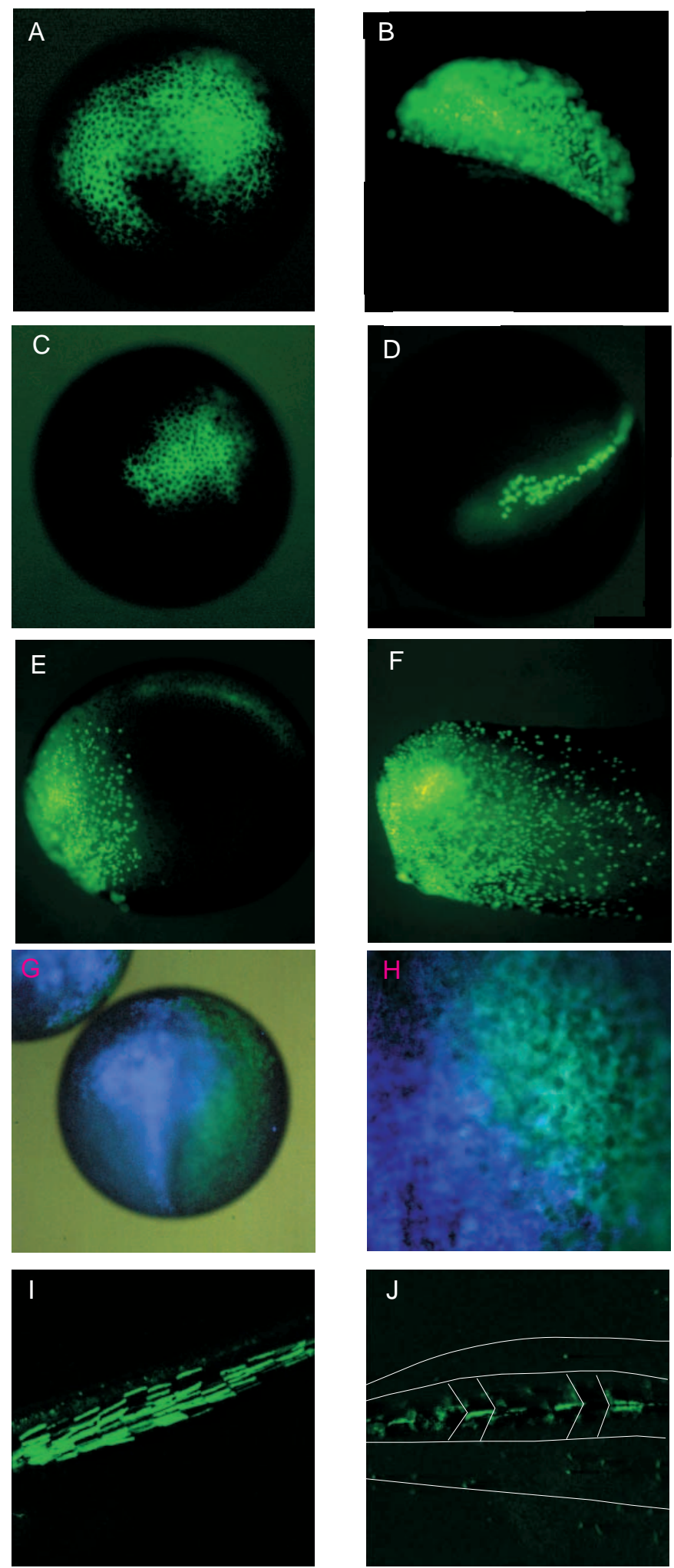
embryo, a disadvantage since, in Xenopus, the pattern of cleavage (and thus the lineage of labelled cells) is by no means identical in all embryos. This constraint does not apply to GFP.RN3, which can be viewed in living cells. We have followed the descendants of single injected blastomeres, by viewing the same living embryos over several days.

When GFP.RN3 mRNA is injected into only one blastomere of a 2-cell egg, the green fluorescent cells are always seen to be dispersed widely on one side of the embryo, but to be virtually or more often completely absent on the other side (Fig. 2B). We therefore see that this marker does not cross cell walls and that there is no detectable mixing of epidermal cells between the two sides of an embryo. The intensity of the fluorescence provides a sensitive test of this conclusion.

We have examined in more detail the lineage of single blastomeres injected at the 32- to 64-cell stage. Blastomeres of the A tier (nearest the animal pole) give rise to groups of cells that are at first localized to a small region of the blastula, but they become progressively intermixed with their neighbours as development proceeds (Fig. 2C-F).

Single cells from the B or C tiers of 32- to 64-cell embryos nearly always contribute to internal cells, especially muscle, but often to some epidermal cells as well. The distribution of cells in muscle has given the surprising result that the GFP.RN3marked cells (from one blastomere) generally contribute to a large number of myotomes, but to only a few muscle cells within each myotome (Fig. 2I-J). Remarkably, we often see an alternating, or at least interspersed, distribution of single muscle cells (Fig. 2J). This suggests that several stem cells are often formed from certain B or C blastomeres, and that these continue
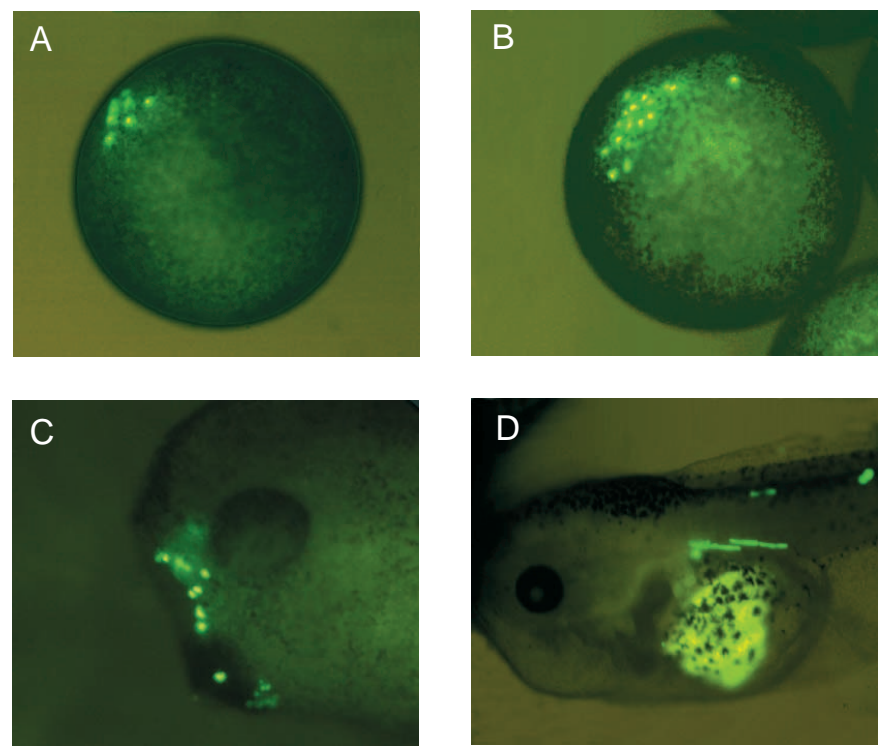

Fig. 3. Transplantation of a single stage-8, GFP.RN3-labelled cell into the blastocoel of an unlabelled stage- 8 host. (A) stage 10; (B) stage 12; (C) stage 38; (D) stage 45. (A-C) Animal cap cell; (D) equatorial cell. In D the intestine appears yellow due to autofluorescence that develops in the tadpole gut. This autofluorescent yellow is unrelated to green fluorescence and is very clearly distinguishable when viewed under the microscope. The yellow spots in the middle of the single green cells in A-C are an artefact of photographic film, and are seen under the microscope as maximum intensity green (but not yellow) fluorescence. to contribute individual cells to many myotomes during tail formation. Thus the muscle cells that comprise a single myotome originate from several early blastomeres.

Blastomeres from the D tier of 32- to 64-cell embryos contribute, as would be expected (Dale and Slack, 1987; Moody, 1987), very largely to the endoderm and gut.

For some experiments, it is helpful to have a second lineage marker that can be used in conjunction with the first, and observed under the same conditions. We have mutated GFP.RN3 to obtain a blue fluorescence (Materials and Methods). Our BFP.RN3 causes cells to emit a blue fluorescence under UV light ( $380 \mathrm{~nm}$ ). Like GFP.RN3, BFP.RN3 can be seen in living embryos, though it is gradually bleached by the UV light. It is not as intense as the GFP, the latter being so strong that it can be seen at the same time as the BFP under $380 \mathrm{~nm}$ light (Fig. 2G,H). BFP can therefore be used as a lineage marker complementary to GFP.RN3, after injection of its mRNA.

\section{Single cell transplantation}

Although not often carried out, the transplantation of single cells to ectopic sites in an embryo can give valuable information on the importance of local cell interactions (Heasman et al., 1984; Kato and Gurdon, 1992). In contrast to lineage studies, which determine the fate of cells surrounded by their natural neighbours in a wholly undisturbed normal development, cell transplantation is able to reveal signalling interactions between cells of a kind that would never be evident by observing normal cell lineages. It has been observed in insects as well as in Xenopus that several cells transplanted as a group will maintain their differentiation (or commitment to differentiate), whereas the same cells transplanted singly will not do so (Cockcroft and Gardner, 1987; Technau, 1987; Stüttem and Campos Ortega, 1991; Gurdon et al., 1993a). Experiments of this kind have led to the concept of the community effect (Gurdon, 1988; Gurdon et al., 1993b).

The markers so far used for such experiments do not permit the transplanted cell and its descendants to be followed during the development of a single living embryo. We have tested the usefulness of our marker for such experiments, by transplanting single GFP.RN3-expressing cells from a stage- 8 embryo into the blastocoel of unlabelled stage- 8 hosts, a procedure described before by Heasman et al. (1984). We find that the descendants of such transplanted cells can be readily seen in living embryos, at least up to tadpole stages (Fig. 3A-D). There is no detectable spread or loss of GFP.RN3 from the descendants of the transplanted cells and their descendants.

Just as we observed in our lineage experiments (above), we find that the progeny of a single transplanted cell at first remain near each other but become widely dispersed and intermingled with other cells during neurula and elongation stages. The distribution of single transplanted cells whose progeny contain muscle cells is of special interest, and permits two conclusions. First, a stage- 8 blastomere often generates both muscle and non-muscle (usually epidermal) progeny; therefore commitment to muscle has not taken place by this stage. Second, we see, as in lineage analyses, that a single stage- 8 blastomere will commonly contribute a single muscle cell to the myotomes of several segments along the axis of a stage- 40 or later tadpole (Fig. 3D). From these kinds of experiments, we see that our GFP.RN3 construct can provide a valuable marker for cell transplantation experiments, as it should also for the continuous observation of migrating germ cells, neural crest cells, etc. 


\section{GFP.RN3 and Eomes expression}

Our initial aim was to use GFP.RN3 as a long lasting marker of ephemeral Eomes expression. When Eomes is ectopically expressed in blastula animal caps, by mRNA injection into 2cell eggs, it can induce almost all known early Xenopus genes and a range of later cell-type specific markers, including those for muscle (K. Ryan, N. Garrett, A. Mitchell and J. B. Gurdon, unpublished). Since Eomes mRNA has disappeared by the stage when muscle markers appear, it is not clear whether muscle is induced in cells that were themselves overexpressing Eomes, or whether those cells signal to other cells to do so.

To answer this question, we injected a mixture of Eomes and GFP.RN3 mRNAs into one of the cells of a 2-cell egg (see legend to Fig. 4). We isolated animal caps, placed two of these face to face with each other to provide a larger conjugate with GFP- and non-GFP-containing regions, and then cultured these conjugates until stage 26, when they were tested for expression of the terminal muscle marker 12/101 (Kintner and Brockes, 1984).

Fig. 4A shows that certain regions of the conjugates have greatly elongated into extensions, which are strongly marked by GFP.RN3 and therefore derive from GFP.RN3- and Eomescontaining cells. This makes it clear that Eomes causes cell elongation, an indication of gastrulation movements characteristic of mesoderm, but not of normal animal cap cells. Subsequent sectioning and antibody staining shows that the regions most strongly labelled by GFP.RN3 are also those that are clearly positive for 12/101 antibody staining (Fig. 4B,C). Although occasional green cells are seen in the $12 / 101$ negative region, there is a high density of GFP.RN3-positive cells in the areas most strongly stained by the muscle marker. We conclude that muscle cell fate is a direct response of cells to a high level of Eomes. We see no evidence that Eomes can induce non-Eomes-containing cells to adopt a muscle fate.

\section{DISCUSSION}

The original aim of this work was to provide a way of marking those cells in which an early regulatory gene has been overexpressed, in order to assess the effect of overexpression on the cell fate. The Xenopus gene Eomes, like many other early developmental genes encoding transcription factors, is expressed only briefly and its transcripts are no longer detectable a few hours after its initial activation, well before most markers of cell fate have appeared. The mRNA for the two fluorescent protein-encoding constructs described here can be mixed with other mRNAs for injection by the usual overexpression procedure used in Xenopus. We suppose that two mixed mRNAs are more likely to be distributed to the same daughter cells than, for example, dextran and mRNA. It would not be difficult to make a dicistronic construct for our fluorescent protein and another gene, such that a single injected mRNA will generate both proteins. In the results reported here, we note that the muscle marker $12 / 101$ is not expressed in all GFP.RN3-containing cells (and therefore in all those cells that previously expressed Eomes); it is expressed only in a coherent group of GFP.RN3-containing cells, a situation reminiscent of the community effect (Gurdon et al., 1993a).

We believe that the GFP.RN3 construct described here may be of general value for other embryological work in Xenopus, for the following reasons: (1) GFP fluorescence is intensely visible in single living cells. The injection of $1 \mathrm{ng}$ of GFP.RN3 mRNA generates an intensity of signal in tail-bud tadpoles 40 times stronger under the fluorescence microscope than the injection of $30 \mathrm{ng}$ of rhodamine-lysinated dextran. Therefore GFP mRNA generates a signal over 1000 times stronger than the traditional rhodamine lineage tracer. (2) At least 2 ng mRNA injected per embryo is not toxic. (3) GFP fluorescence can be seen 1.5 hours after injection. (4) There is no significant autofluorescence when viewing GFP.RN3. (5) It is not bleached by the $480 \mathrm{~nm}$ absorption wavelength. (6) The protein is very stable.
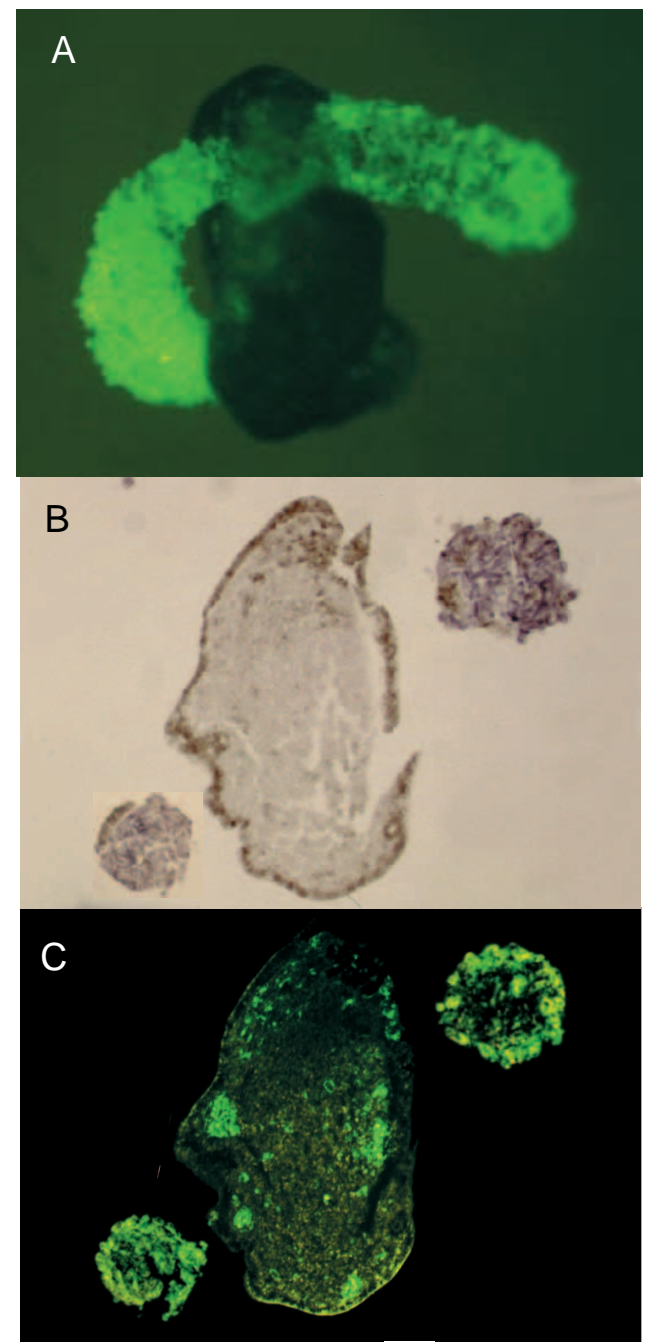

Fig. 4. Use of GFP.RN3 to determine the fate of cells overexpressing Eomes. 2-cell embryos were injected with a mixture of $1 \mathrm{ng}$ GFP.RN3 mRNA and 2 ng Eomes mRNA into one blastomere at the 2-cell stage. This was done in order to achieve animal caps of which only part was induced to form muscle, the rest serving as a no-muscle control. Embryos were cultured to stage 8, when animal caps were removed and placed face to face in pairs, and cultured to control stage 26. (A) An animal cap double conjugate showing GFP.RN3

fluorescence in tissue projections caused by Eomes overexpression.

(B) Transverse section of conjugate in A stained with 12/101 muscle specific antibody; (C) same sample under green fluorescence. Note that the strongly fluorescent extensions (A and $\mathrm{C}$ ) are heavily stained with the muscle antibody (B), but that the central part of the conjugate (not extended) contains few GFP.RN3-containing cells and no 12/101 muscle antibody staining. The dark periphery of the central body is attributable to pigment and not to antibody staining. 
(7) GFP.RN3 is evenly distributed in cells descended from the region of egg injection. (8) This GFP.RN3 withstands traditional histological procedures, although with reduced intensity.

These qualities should enable this marker to be used to answer a number of important questions concerning vertebrate cell lineage. The original work describing compartments in Drosophila and the restrictions they place on the spread of embryonic cell descendants (Morata and Lawrence, 1975) depended not only on the use of Minute mutant cells (Simpson and Morata, 1981), but also on the availability of a longlasting cell lineage marker (reviewed by Lawrence and Struhl, 1996). Further work with our GFP.RN3 and BFP markers could reveal the extent of compartment-like restrictions in vertebrate development.

The results we describe here, in which a single embryo cell contributes only one or a few muscle cells to many segments, suggests that several equatorial cells of a 32-cell embryo contribute muscle-forming stem cells; these stem cells then generate muscle cells continuously as successive segments are formed. A similar conclusion was reached for chick development by Primmett et al. (1989) using ${ }^{3} \mathrm{H}$-thymidine pulse-labelling of somites, and by Selleck and Stern (1991) using DiI-labelling of small groups of future notochord cells (see Stern et al., 1992).

We thank F. Stennard and A. Mitchell for their help with some technical procedures, and A. Brand and C. Davidson for pointing out the presence of the $\mathrm{H} 25 \mathrm{Y}$ mutation in GFP.RN3. M. Z.-G. thanks EMBO for a Long Term Research Fellowship. This work was supported by a Cancer Research Campaign programme grant SP $2184 / 0101$ to J. B. Gurdon.

\section{REFERENCES}

Chalfie, M., Tu, Y., Euskirchen, G., Ward, W.W. and Prasher, D.C. (1994). Green fluorescent protein as a marker for gene expression. Science $\mathbf{2 6 3}, 802$ 805 .

Cockroft, D.L. and Gardner, R.L. (1987). Clonal analysis of the developmental potential of 6th and 7th day visceral endoderm cells in the mouse. Development 101, 143-155.

Cormack, B.P., Valdivia, R.H. and Falkow, S. (1996). FACS-optimized mutants of the green fluorescent protein (GFP). Gene 173, 33-38.

Dale, L. and Slack, J.M.W. (1987). Fate map for the 32-cell stage of Xenopus laevis. Development 99, 527-551.

Dawid, I.B. (1994). Intercellular signalling and gene regulation during early embryogenesis of Xenopus laevis. J. Biol. Chem.. 269, 6259-6262.

Gardner, R.L. and Lawrence, P.A. (ed.) (1986). Single Cell Marking and Cell Lineage in Animal Development. The Royal Society, London.

Gimlich, R.L. and Braun, J. (1985). Improved fluorescent compounds for tracing cell lineage. Dev. Biol. 109, 509-514.

Gurdon, J.B. (1988). A community effect in animal development. Nature 336, $772-774$.

Gurdon, J.B., Tiller, E., Roberts, J. and Kato, K. (1993a). A community effect in muscle development. Current Biol. 3, 1-11.

Gurdon, J.B., Lemaire, P., and Kato, K. (1993b). Community effects and related phenomena. Cell 75, 831-834.

Heasman, J., Wylie, C.C., Hausen, P. and Smith, J.C. (1984). Fates and states of determination of single vegetal pole blastomeres of Xenopus laevis. Cell 37, 185-194.

Heim, R., Prasher, D.C. and Tsien, R.Y. (1994). Wavelength mutations and posttranslational autoxidation of green fluorescent protein. Proc. Natl Acad. Sci. USA 91, 12501-12504.

Heim, R., Cubitt, A.B. and Tsien, R.Y. (1995). Improved green fluorescence [letter]. Nature 373, 663-664.

Heim, R. and Tsien, R.Y. (1996). Engineering green fluorescent protein for improved brightness, longer wavelengths and fluorescence resonance transfer. Curr. Biol. 6, 178-182.

Jacobson, M. (1983). Clonal organization of the central nervous system of the frog. III. Clones stemming from individual blastomeres of the 128-, 256-, and 512-cell stages. J. Neurosci. 3, 1019-1038.

Jacobson, M. (1985). Clonal analysis and cell lineages of the vertebrate central nervous system. Annu. Rev. Neurosci. 8 71-102.

Jacobson, M. and Hirose, G. (1981). Clonal organization of the central nervous system of the frog. II. Clones stemming from individual blastomeres of the 32- and 64-cell stages. J. Neurosci. 1, 271-284.

Kato, K. and Gurdon, J.B. (1992). Single-cell transplantation determines the time when Xenopus muscle precursor cells acquire a capacity for autonomous differentiation. Proc. Natl Acad. Sci. USA 90, 1310-1314.

Kintner, C.R. and Brockes, J.P. (1984). Monoclonal antibodies identify blastemal cells derived from dedifferentiating muscle in newt limb regeneration. Nature 308, 67-69.

Lawrence, P.A. and Struhl, G. (1996). Morphogens, compartments, and pattern: lessons from Drosophila? Cell 85, 951-961.

Lemaire, P., Garrett, N. and Gurdon, J.B. (1995). Expression cloning of Siamois, a new Xenopus homeobox gene expressed in dorsal vegetal cells of blastulae and capable of inducing a complete secondary axis. Cell 81, 85-94.

Moody, S. (1987). Fates of the blastomeres of the 32-cell-stage Xenopus embryo. Dev. Biol. 122, 300-319.

Morata, G. and Lawrence, P.A. (1975). Control of compartment development by the engrailed gene in Drosophila. Nature 255, 614-617.

Nakamura, O. and Kishiyama, K. (1971). Prospective fates of blastomeres at the 32 cell stage of Xenopus laevis embryos. Proc. Japan Acad. 47, 407-412.

Nakamura, O., Takasaki, H. and Nagata, A. (1978). Further studies on the prospective fates of blastomeres at the 32 cell stage of Xenopus laevis embryos. Med. Biol. 56, 355-360.

Niehrs, C. and De Robertis, E.M. (1991). Ectopic expression of a homeobox gene changes cell fate in Xenopus embryos in a position-specific manner. EMBO J. 10, 3621-3629.

Peters, K.G., Rao, P.S., Bell, B.S. and Kindman, L.A. (1995). Green fluorescent fusion proteins: powerful tools for monitoring protein expression in live zebrafish embryos. Dev. Biol. 171, 252-257.

Prasher, D.C., Eckenrode, V.K., Ward, W.W. Prendergast, F.G. and Cornier, M.J. (1992). Primary structure of the Aequaria victoria green fluorescent protein. Gene 111, 229-233.

Primmett, D.R.N., Norris, W.E., Carlson, G.J., Keynes, R.J. and Stern, C.D. (1989). Periodic segmental anomalies induced by heat shock in the chick embryo are associated with the cell cycle. Development 105, 119-130.

Selleck, M.A.J. and Stern, C.D. (1991). Fate mapping and cell lineage analysis of Hensen's node in the chick embryo. Development 112, 615-626.

Simpson, P. and Morata, G. (1981). Differential mitotic rates and patterns of growth in compartments in the Drosophila wing. Dev. Biol. 85, 299-308.

Slack, J.M.W. (1983). Cell lineage labels in the early amphibian embryo. BioEssays 1, 5-8.

Smith, J.C. (1993). Mesoderm-inducing factors in early Vertebrate development. EMBO J. 12, 4463-4476.

Smith, J.C., Dale, L. and Slack, J.M.W. (1985). Cell lineage labels and region-specific markers in the analysis of inductive interactions. J. Embryol. Exp. Morph. 89 Supplement 317-331.

Stern, C.D., Hatada, Y., Selleck, M.A.J. and Storey, K.G. (1992). Relationships between mesoderm induction and the embryonic axes in chick and frog embryos. Development Supplement 151-156.

Stüttem, I. and Campos-Ortega, J.A. (1991). Cell commitment and cell interactions in the ectoderm of Drosophila melanogaster. Development Supplement 2, 39-46.

Tannahill, D., Bray, S., and Harris, W.A. (1995). A Drosophila E(spl) gene is neurogenic in Xenopus - a green fluorescent protein study. Dev. Biol. 168 694-697.

Technau, G.M. (1987). A single cell approach to problems of cell lineage and commitment during embryogenesis of Drosophila melanogaster. Development 100, 1-12.

Wu, G.Y., Zou, D.J., Koothan, T. and Cline, H.T. (1995). Infection of frog neurons with vaccinia virus permits in vivo expression of foreign proteins. Neuron 14, 681-684. 